\title{
PANDEMIA Y CONVIVENCIA ESCOLAR: INCERTIDUMBRES Y RETOS
}

\author{
José María Avilés Martínez \\ Universidad de Valladolid, España
}

\begin{abstract}
Resumo: Cuando se estudia o trabaja en educación en general, y en convivencia escolar en particular, siempre se hace gala de aprovechar las crisis, sean estas de la naturaleza que sean, para considerarlas oportunidades educativas de las que aprender y puntos de partida para mejorar el futuro de las personas que están bajo responsabilidad. La historia de la educación ha ido sumando oportunidades que la evolución de la propia sociedad le ofrecía para construir esas respuestas. En este sentido, la crisis del Covid-19, de escala planetaria, es una oportunidad única que ha puesto a prueba muchas cosas en nuestra sociedad colectivamente, y en cada una de las personas que la componen, para paliar e intentar resolver las múltiples consecuencias derivadas de la emergencia sanitaria en la ciudadanía, en su convivencia social, su bienestar emocional y su propia salud. La educación y lo que sucede en las escuelas no se ha quedado al margen y han sido exigidas, y de qué manera, para ofrecer soluciones adaptativas durante la pandemia y también respuestas transformadoras para el futuro, analizando y reflexionando sobre lo éramos-teníamos-hacíamos educativamente y lo que queremos ser-tener-hacer en la escuela cuando las sucesivas olas epidémicas cesen y sigamos construyendo nuestra escuela.
\end{abstract}

Por tanto, en un primer momento del análisis es necesario detenerse sobre el escenario educativo y de relaciones interpersonales y de convivencia, para examinar la evolución y condicionantes que estaban activos antes de la pandemia, los que han estado actuando durante su evolución y los que previsiblemente ya se apuntan como retos que abordar cuando dejemos atrás la crisis sanitaria y social. Esto va a ayudar a avanzar, pensando, que si se es capaz de tomar nota de las dificultades que se han encontrado para afrontar estos momentos desde el punto de vista educativo, se tendrán más argumentos para organizar mejor las respuestas posteriores.

Si se piensa en la educación, en qué relaciones y en qué convivencia había en nuestras escuelas antes de la crisis, la reflexión ayudará a identificar sus debilidades y el potencial que ha dejado al descubierto, ya que eran las herramientas educativas con las que se ha contado para afrontar esta emergencia. Por otra parte, también es necesaria la reflexión sobre cómo se está afrontando la educación y la convivencia escolar mientras dura la crisis porque los defectos o desajustes de respuesta pueden iluminar para seguir abordando sucesivas olas y confinamientos que todavía hoy son posibles en muchos lugares. Y finalmente, es fundamental hacerlo porque la experiencia vivida está enseñado muchas cosas sobre las formas de relación interpersonal, la ciberconvivencia y sobre formas de violencia que es necesario abordar desde múltiples

\footnotetext{
1 Doutor em psicologia da Educação, professor da Universidad de Valladolid, España. E-MAIL: martinez@valladoid.es.com. ORCID: https://orcid.org/0000-0001-9179-6427

Revista Tópicos Educacionais, Pernambuco, v. 27, n. 01, p. 01-22, 2021. ISSN: 2448-0215. https://periodicos.ufpe.br/revistas/topicoseducacionais/index Dossiê "Conflitos, violências, bullying na escola: problemas da convivência potencializado pela pandemia?" DOI: 10.51359/2448-0215.2021.250402
} 
perspectivas. Ello supondrá poner en valor nuevos retos y un trabajo educativo renovado que se tendrá que abordar y organizar en la escuela y en la comunidad educativa si se quiere que algo cambie tras lo que nos ha sucedido como sociedad.

En definitiva, problemas tan globales como el cambio climático, la violencia estructural, el crecimiento insostenible, el racismo o la xenofobia, la migración forzada, la violencia de género, ... no son ajenos a la educación, son solo algunos ejemplos que ahora forman parte impostergable del compromiso de transformación social de la escuela, a cuyo combate se tiene que sumar en su propio escenario traspandémico, de cambio de relaciones interpersonales y convivencia más justa y positiva en que otros valores se postulen. Sin duda, no va a ser fácil, nadie dijo que lo fuera a ser y la escuela viene demostrando desde siempre que no lo es, pero es necesario no cejar para vencer las resistencias que cualquier cambio social produce, ya que la escuela en muchas ocasiones también colabora en reproducir lo injusto del sistema social que hace infelices, víctimas y vulnerables a las personas que lo habitan. Y eso también se aprende y perpetúa de continuo en el cotidiano de los contextos escolares, cuando en su seno se vuelven a reproducir relaciones machistas, abusivas, injustas, violentas o discriminatorias. Luchar contra ello es un mandato moral innegable e impostergable que quienes educan tienen ante sí tras la crisis que se está viviendo. Ojalá la escuela pueda combatirlo para empezar a desmontar la anestesia individual y social ante la falta de respuesta al abuso, la aceptación y desensibilización ante las desigualdades e injusticias o las irresponsabilidades cívicas frente a las arbitrariedades políticas, económicas o de las mayorías (Reardon, 2020). Las soluciones que se necesitan pasan indefectiblemente por este itinerario de reflexiones, compromisos y cambios que se han de enmarcar en proyectos educativos comunes y que quienes educan no pueden eludir.

\section{LA HERENCIA RECIBIDA: LA CONVIVENCIA DURANTE LA PANDEMIA. VULNERABILIDADES, DESIGUALDADES Y RETOS.}

Cuando surge la pandemia la escuela tiene que abordarla con las herramientas de que dispone en ese momento, en sus fortalezas y debilidades. Ante una amenaza semejante las

Revista Tópicos Educacionais, Pernambuco, v. 27, n. 01, p. 01-22, 2021. ISSN: 2448-0215. https://periodicos.ufpe.br/revistas/topicoseducacionais/index Dossiê "Conflitos, violências, bullying na escola: problemas da convivência potencializado pela pandemia?" DOI: $10.51359 / 2448-0215.2021 .250402$ 
respuestas adaptativas han sido muy diferentes, pero todas se han caracterizado por ser reacciones improvisadas al principio y progresivamente más ajustadas después. En cualquier caso, llevadas a la práctica en un clima de incertidumbre frente a la evolución de la crisis y de un compromiso certero en asegurar los procesos básicos educativos y de convivencia por parte de los educadores. Sin embargo, la realidad impone que la escuela era la misma el día antes de la pandemia y el de después. Los cambios y adaptaciones se han leído más tarde y han estado condicionados no solo por la propia crisis sanitaria sino por la inercia evolutiva que la propia escuela arrastraba, con sus déficits y potencialidades.

En el debe, la escuela que se ha heredado en la pandemia ha venido reproduciendo los valores más identitarios del propio rumbo económico y social vigente y que han explicado su utilidad estratégica para lo que hemos denominado la felicidad sistémica (Avilés, 2020a). Sin embargo, estos valores, sostenedores del estatus quo actual, ya hace tiempo que se venían mostrando insostenibles y son exponentes de una desigualdad económica y social que la crisis ha desnudado en nuestra escuela, ante nuestras caras, mostrando la vulnerabilidad y desigualdad de condiciones y derechos. Son valores como la sobreproducción, la especulación, el crecimiento insostenible, la cultura del éxito individual frente al grupo social o la independencia personal de los derechos frente a la interdependencia colectiva de los deberes. Si algo ha cuestionado más palmariamente la crisis sanitaria ha sido esto último. La escuela ha formado exclusivamente para el éxito individual y ha logrado consumidores perfectos aunque ciudadanos imperfectos, colocándolos como sujetos de negocio y no como sujetos de derechos (Avilés, Aragao, Menin, Nunes y Braga, 2020). Esta reproducción tiene correlato específico en el plano de las relaciones interpersonales y de convivencia en la propia escuela en que en muchos contextos venían primado valores individuales frente a colectivos, elementos de abuso frente a situaciones y figuras vulnerables, discriminadas y/o violentadas o contextos de carencias y déficits de derechos frente a decisiones economicistas o de posibilismo práctico. Son algunos de los contravalores frente a los que, por otra parte también, se venían fomentado en muchas escuelas algunas propuestas educativas que promovían el respeto, la tolerancia, la solidaridad y la justicia en el ideario y en las prácticas educativas en la escuela.

De otro lado, también han marcado la respuesta educativa durante la crisis la rígida configuración existente del propio currículo escolar y sus exigencias normativas y de peso de los contenidos académicos frente a otros más emocionales, emocionantes y experienciales (Avilés,

Revista Tópicos Educacionais, Pernambuco, v. 27, n. 01, p. 01-22, 2021. ISSN: 2448-0215. https://periodicos.ufpe.br/revistas/topicoseducacionais/index Dossiê "Conflitos, violências, bullying na escola: problemas da convivência potencializado pela pandemia?" DOI: $10.51359 / 2448-0215.2021 .250402$ 
2020a), además de la configuración del propio sistema educativo, estructuralmente poco flexible y menos adaptable. Esto ha supuesto una resistencia a la adaptación necesaria, manifiesta en los propios gestores y administradores de las escuelas, trasmitida a docentes, que en muchas escuelas ha dejado un tanto de lado el plano emocional y asistencial, lo que ha agravado, si cabe más, vulnerabilidades explícitas en perfiles de alumnado con necesidades sociales, familiares o educativas. Es decir, muchas escuelas no han sido lo suficientemente versátiles curricularmente para responder adaptativamente a la diversidad de necesidades vitales que se planteaban en su contexto. Esto ha incidido en el avance del alumnado en su conexión académica. Muchos de ellos tenían la escuela como su única oportunidad para el progreso personal y salvavidas de vulnerabilidad. Además, ha afectado al ya escaso y heredado nivel de participación de la comunidad educativa en las escuelas. La pobre presencia real y colegiada existente en la toma de decisiones en los centros de familias, profesorado y alumnado se ha mostrado un inconveniente para el abordaje de la adaptación a los cambios exigidos por la pandemia. Se ha padecido un sistema de relaciones participativas entendido como una fórmula delegada de gestión a los equipos gestores y directores, que durante la pandemia han estado mediatizados por órdenes, en ocasiones contradictorias y poco realistas, que con frecuencia, de forma inadecuada y evasiva, abusaban de la tan manida 'autonomía de los centros'.

En este sentido, este déficit de empoderamiento en la toma de decisiones ya se manifestaba con el alumnado en la escuela de la que venimos. Y cuando se ha preguntado al alumnado sobre cómo se sintieron durante el confinamiento se volvió a replicar (Tonucci, 2020). Ellos han percibido una respuesta de la escuela demasiado réplica de lo presencial e infraemocional, que no les ha ayudado a resolver sus principales incertidumbres, sin duda favorecida por la presión y el estrés resolutivo a que el profesorado se ha visto sometido también para solventar contenidos, en una escuela, que hasta entonces, tenía una presencia mucho más analógica y presencial que digitalizada y a distancia. Esto habla, de nuevo, de un déficit de respuesta emocional que la escuela no ha podido proporcionar a su alumnado durante la crisis y que todavía no tenía el espacio que le corresponde en una escuela que debía caracterizarse por atender la seguridad individual y colectiva, el ajuste social, la salud emocional y el bienestar personal de su alumnado por encima de otras exigencias. Y ello requiere espacios de participación democrática en su seno por parte de todos los miembros de la comunidad educativa, familias, alumnado y profesorado.

Revista Tópicos Educacionais, Pernambuco, v. 27, n. 01, p. 01-22, 2021. ISSN: 2448-0215. https://periodicos.ufpe.br/revistas/topicoseducacionais/index Dossiê "Conflitos, violências, bullying na escola: problemas da convivência potencializado pela pandemia?" DOI: $10.51359 / 2448-0215.2021 .250402$ 
Sin embargo, la emergencia vivida también ha puesto al descubierto nuestras fortalezas como escuela presencial. Hemos sido más conscientes, si cabe aún, del papel de cohesión social que ha tenido la escuela pública en nuestra sociedad como elemento asegurador de marcos de igualdad de oportunidades y como compensador y ascensor social para reducir las desigualdades. En algunos casos, se ha mostrado como el único factor de ayuda y sostén para muchos, los más vulnerables, en situaciones por ejemplo, de alimentación, protección, supervisión y guía o prevención del abandono temprano.

A algunos alumnos y alumnas les ha servido para mantener el vínculo que les permite seguir conectados al sistema con mínimas posibilidades de inserción social o laboral. Esta vinculación escolar también antes se ha revelado como un potencial de la institución en los procesos de socialización y encuentro del alumnado a través de los momentos de participación y convivencia, posibilitando los procesos de integración social o de enriquecimiento de la red de relaciones interpersonales, tan necesarias para la salud y el bienestar personal entre niños, niñas y adolescentes. Y es en esos momentos de convivencia presencial donde la escuela ofrece otro de sus potenciales, a través de herramientas emocionales y de pensamiento para favorecer el aprendizaje práctico de respuestas ante situaciones de conflictividad o abuso, mediante posicionamientos ante el dominio o la violencia, expresando valores deseables de respeto a la diversidad, por la igualdad o la tolerancia y la solidaridad. En definitiva, también se ha partido de una escuela que era modelo de valores críticos y alternativos al pensamiento dominante.

Sin embargo, si algo ha supuesto la pandemia para las relaciones interpersonales y sociales ha sido un sistema de distanciamiento que ha trasmitido un mensaje simbólico subyacente que ha percutido de forma directa en la línea de flotación de la convivencia. La figura de la otra persona como fuente de riesgo de contagio con recomendaciones de distancia, alejamiento y protección, armando un discurso en el que las otras personas son potenciales amenazas por su asintomatismo, por sus síntomas o por su enfermedad, por lo que lo que parecía más prudente era el alejamiento personal y la configuración del otro como potencial peligro. Algo demoledor, también en la escuela, para construir esos vínculos sociales y expresar el contacto cercano y el disfrute de la propia convivencia como se viene trabajando y practicando. Algunas de estas potencialidades de la vida escolar durante la pandemia se han visto eliminadas o se han limitado y/o pospuesto durante los períodos más duros de confinamiento como medidas de distanciamiento impuestas. Sin embargo, los procesos de enseñanza-aprendizaje han debido proseguir sin la

Revista Tópicos Educacionais, Pernambuco, v. 27, n. 01, p. 01-22, 2021. ISSN: 2448-0215. https://periodicos.ufpe.br/revistas/topicoseducacionais/index Dossiê "Conflitos, violências, bullying na escola: problemas da convivência potencializado pela pandemia?" DOI: 10.51359/2448-0215.2021.250402 
presencialidad de forma digital. Y esto ha mostrado durante estos períodos de confinamiento las carencias que el propio sistema educativo en toda su extensión tenía para seguir dando respuesta, así como la inquebrantable capacidad de sus integrantes para persistir en el objetivo.

La situación ha puesto de manifiesto unas brechas que han agravado la desigualdad, violencias en el acceso, el desarrollo y el seguimiento que los más vulnerables ya tenían en el sistema. Brechas digital, social, cultural, económica, informativa, asistencial o de aprendizaje. En el caso de la escuela, los más vulnerables y diversos han perdido la equidad que requerían porque la digitalización forzada de la educación les ha colocado en desigualdad un listón que no podían saltar. No tanto por culpa de la digitalización sino más bien por el rosario de carencias que la propia escuela y la educación en relación a las tecnologías, aún hoy, sigue teniendo al respecto. Por tanto, la pandemia se ha revelado como un catalizador para repensar la tecnología y su uso en la escuela (Avilés et al., 2020). No solo, que también, porque existieran más o menos dispositivos, tampoco solo, que también, porque hubiera más o menos puntos de conexiones o plataformas digitales, sino, sobre todo, porque aún la metodología didáctica, la forma de dar la clase, no se ha 'digitalizado'. En muchos casos se ha pretendido seguir dando clase de la misma forma que antes y el peso de la memoria respecto a los contenidos o el absolutismo de las fuentes únicas (de texto) ha predominado como elementos de seguridad de los y las docentes. También ha habido muy honrosos profesionales que son cada vez más que han incorporado otras formas. Y esto tiene que ver con que aprender a hacer, aprender a pensar o aprender a buscar información requiere competencias que enseñen a discriminar lo principal de lo accesorio, lo nuclear de lo superfluo, lo falso de lo cierto, lo primario de lo derivado,... en definitiva, una metodología que tanto presencialmente como digitalmente tiene que ser diferente, más innovadora, creativa, formativa, planificada, crítica, disonante, independiente, confiada, evaluable, ... y menos memorística, aburrida, previsible y unidireccional.

Sin embargo, el uso de los dispositivos y lo digital es y está en la vida de las y los escolares, forma parte de sus emociones y de su convivencia dentro y fuera de la escuela. Sus relaciones interpersonales se dilucidan hoy en las redes, sus emociones, su imagen, su estatus social hoy también es digital. La ciberconvivencia es un nuevo reto educativo que debe abordarse sin dilación en los planes de las escuelas (Avilés, 2021), pues si no se hace de forma adecuada y educativa, tanto las familias como los docentes y la escuela llegaremos tarde a ese escenario en el que intervenir.

Revista Tópicos Educacionais, Pernambuco, v. 27, n. 01, p. 01-22, 2021. ISSN: 2448-0215. https://periodicos.ufpe.br/revistas/topicoseducacionais/index Dossiê "Conflitos, violências, bullying na escola: problemas da convivência potencializado pela pandemia?" DOI: $10.51359 / 2448-0215.2021 .250402$ 
Sin embargo, la pandemia nos ha dado la oportunidad para hacer otras cosas y de otra forma. Referencias recientes (CLAIP, 2020; Moreira, 2020; Reardon, 2020; Trujillo et al., 2020) identifican las opiniones de los sectores sociales y de la comunidad educativa reflejando sus vivencias sociales, laborales y vitales en relación a los procesos educativos durante este período.

El alumnado reconoce haber aprendido muchas cosas diferentes que les ha enfrentado a otras competencias (hacer comidas, quedarse solos, encajar una enfermedad,... han tenido más tiempo para estar con sus familias que les ha permitido conocerlas y disfrutar de ellas más, aunque también han sentido la ausencia física de sus amistades y cierta falta de comprensión de lo que les sucedía, especialmente en los más pequeños. Sin embargo, en el plano escolar lo más sufrido para ellos ha sido la excesiva carga de deberes y la reproducción automática de las clases presenciales en las pantallas. Entre los más vulnerables se refleja una pérdida de conexión escolar y falta de motivación y vínculo entre lo más significativo.

Las familias han acusado el mucho el tiempo que han pasado con sus hijos e hijas tanto en lo bueno (más compartir y mejor acompañamiento) como en lo negativo (excesivo estrés, mucho peso escolar del que hacerse cargo también para ellos, que a algunos les ha hecho reproducir íntegramente los contenidos curriculares) y muchas dificultades para la conciliación. Sin embargo, entre las más vulnerables, es la falta de medios, pérdida de seguimiento escolar y riesgo en sus hijos e hijas de abandono, lo más común.

El profesorado reconoce el estrés que le ha producido el tremendo esfuerzo que, en general, ha tenido que hacer para adaptarse de un día para otro a la enseñanza a distancia y mantener metodológicamente ese sistema desde el punto de vista motivacional con el alumnado, así como las tareas de su evaluación y supervisión de forma personalizada. Estas dificultades de seguimiento han sido especialmente mayores con el alumnado más vulnerable, que en algunos casos ha estado perdido durante este período. Es significativa la queja del profesorado respecto a su Administración Educativa de la que no reconoce apoyo, y su recurso excesivo a la 'autonomía' cuando no era capaz de dar respuestas concretas. Igualmente, se reconoce un menor control individual en la enseñanza on line que en la situación de presencialidad y la fuerte y extensa carga académica de contenidos que han manejado con el alumnado.

De estos datos parece que se desprende la necesidad de incorporar significativas mejoras en cuanto al acceso y uso de la tecnología y en el uso adecuado de plataformas comunitarias y de fuentes diversas de información y su integración en la práctica docente, y una

Revista Tópicos Educacionais, Pernambuco, v. 27, n. 01, p. 01-22, 2021. ISSN: 2448-0215. https://periodicos.ufpe.br/revistas/topicoseducacionais/index Dossiê "Conflitos, violências, bullying na escola: problemas da convivência potencializado pela pandemia?" DOI: $10.51359 / 2448-0215.2021 .250402$ 
reducción significativa de los currículos en situaciones como la educación distanciada, ciñéndolos a lo básico para avanzar. Parece necesario igualmente aumentar el apoyo socioemocional que se presta a todo el alumnado, pero en especial, durante situaciones de distanciamiento, en concreto, al más vulnerable y en riesgo de exclusión. En este sentido, la escuela tiene que incorporar cada vez más intencional y curricularmente una pedagogía de cuidados (Avilés, 2020b) y el trabajo sobre las ecohabilidades (Avilés et al., 2020a) que están en la base de muchas competencias educativas, profesionales y sociales, la sensibilidad, la flexibilidad, el control de la ira, la honradez, la capacidad para buscar soluciones y resolver conflictos, la coherencia, la sinceridad, el manejo del tiempo o la creatividad.

Se hacen necesarios estos cambios porque es muy probable que todavía tengamos que alternar períodos de cierre y apertura, y de enseñanza, combinando la presencia y a distancia en sucesivas olas de contagios, en esta o en otras pandemias. De ello se deduce que la escuela debe hacerse más versátil y ser capaz de moverse con tiempo entre la incertidumbre y la liquidez (Bauman, 2013), incorporando herramientas que le permitan ser más participativa y sanadora con sus protagonistas, al tiempo que más digital y diversificada. Esa condición de versatilidad tiene que hacer a la escuela más dispuesta a la adaptación, y estructural y organizativamente, más adaptable. El currículo se tiene que nuclearizar en lo básico; con las familias se ha de ser más pedagógico, para ayudarles a gestionar la colaboración educativa; en momentos emocionalmente tan complicados se ha de focalizar la atención de las escuelas a los procesos más vitales del alumnado, sus emociones y preocupaciones, por lo que habrá que cambiar las costumbres de los docentes reduciendo su focalización academicista; esa ralentización inevitablemente hará que se tenga que renunciar a parte de los programas y combinar los aprendizajes con otro tipo de competencias en casa. Sin duda, también en la escuela habrá que abordar de forma estructural, participada e intencional la educación de la ciberconvivencia con pautas educativas compartidas por los sectores de la comunidad educativa que prevenga situaciones de conflicto, abuso o violencia digital y fomente las buenas prácticas en las redes sociales y el disfrute y el aprovechamiento positivo de su uso. En definitiva, una educación más acompañada, guiada y tutorial que atienda las desigualdades y la diversidad que la distancia acusa.

Sin duda, todos ellos son retos complejos e importantes para desarrollar acciones educativas en las escuelas y para reforzar e incrementar el bienestar de sus protagonistas, pero que exigen ya poner en marcha en ellas procesos para un cambio que resulta necesario con el fin de

Revista Tópicos Educacionais, Pernambuco, v. 27, n. 01, p. 01-22, 2021. ISSN: 2448-0215. https://periodicos.ufpe.br/revistas/topicoseducacionais/index Dossiê "Conflitos, violências, bullying na escola: problemas da convivência potencializado pela pandemia?" DOI: $10.51359 / 2448-0215.2021 .250402$ 
poder dar respuesta a sus necesidades más imperiosas. Dado que el espacio que se brinda aquí es reducido para el desarrollo extendido de todos los tópicos, en este texto se abordarán dos que consideramos prioritarios y fundamentales, temáticas que por su relevancia es urgente acometer de forma intencional y vertebrada en la escuela, ya que inciden de forma directa y vitar sobre la salud emocional de los escolares y su convivencia en la escuela y fuera de ella.

\section{ASIGNATURA PENDIENTE DE LA CONVIVENCIA ESCOLAR: LA PEDAGOGÍA DE LOS CUIDADOS.}

La crisis sanitaria que se está viviendo a nivel global está incidiendo en todos los planos vitales de nuestra sociedad, y la escuela como reflejo de ella, reproduce en su seno y con sus protagonistas toda la gama de incertidumbres, dudas y emociones posibles ante esta experiencia. Como se ha señalado, de entre todas las necesidades detectadas en la escuela pandémica los estudios señalan preferente y prioritariamente una como sentida por los distintos sectores de la comunidad educativa (Trujillo et al., 2020): el impacto emocional que las situaciones de confinamiento y distanciamiento social pueden llegar a provocar en los menores, con las consiguientes acciones de intervención necesarias (respuestas minimizadoras del impacto y estimuladoras de normalización) por parte de los educadores que están responsabilizados de ellos. Dependiendo del rango de edad, este impacto se dirige a distintas facetas del desarrollo bio-psicosocial, pero en cualquier caso, el alumnado de las escuelas se está viendo afectado desde su socialización hasta su necesidad de contacto físico. De igual forma, la escuela debe discriminar estas necesidades y de forma intencional, organizada y programada debe dar una respuesta diferenciada y compensadora desde el punto de vista profesional a estas afectaciones que inciden directa y globalmente sobre la evolución psicosocial del alumnado en los distintos momentos de su desarrollo.

Ciertamente, estas respuestas, al inicio de la pandemia tuvieron que darse improvisadas, pero en el punto en que se encuentra ahora, ha habido tiempo para discriminar necesidades, valorar el impacto, seleccionar herramientas y aplicar acciones que ayuden a quienes han sufrido y sufren los daños para ayudarles a avanzar para subsanarlos. Y esto debe hacerse de manera individual, cada uno de los profesionales desde su perspectiva y en su entorno más inmediato, pero especialmente se debe articular de forma colectiva, en el seno de la comunidad educativa, para conseguir que la escuela sea un espacio que cure y sane, en la medida de sus

Revista Tópicos Educacionais, Pernambuco, v. 27, n. 01, p. 01-22, 2021. ISSN: 2448-0215. https://periodicos.ufpe.br/revistas/topicoseducacionais/index Dossiê "Conflitos, violências, bullying na escola: problemas da convivência potencializado pela pandemia?" DOI: $10.51359 / 2448-0215.2021 .250402$ 
posibilidades, todas esas secuelas que la pandemia ha dejado y está aún dejando y que se llaman pérdidas, miedos, incertidumbres, soledades, desesperanzas, incomunicación, desvinculación, abandono, ... Porque si algo de bueno tiene la escuela es que representa una institución y alberga un espacio común en el que todos y cada uno de sus miembros se siente nombrado (singularizado y personalizado), reconocido (ubicado) y atendido (protegido) y del que espera transitar por un proyecto común que es y tiene carácter colectivo. Y es, como nos ha enseñado esta pandemia, de forma colectiva como podremos salir de una situación tan dañina como esta, como colectividad. Pero no solo desde el punto de vista epidemiológico, sino también desde el punto de vista emocional. En la escuela debemos interiorizar que colectivamente somos también más fuertes, que el apoyo mutuo se hace imprescindible y que será juntos como podamos también restañar las heridas emocionales que la pandemia nos está dejando.

Y es que esta situación de crisis también brinda a la escuela (profesorado, familias y alumnado) la oportunidad para construir respuestas hasta ahora distintas o menos atendidas, que ayuden a levantar una escuela diferente a la que se tenía. Una escuela que se preocupa por atender las necesidades emocionales del alumnado, sus miedos, sus expectativas, sus debilidades, sus diferencias, ... alejadas en muchos casos del baremo evaluador de los curricula o de las exigencias administrativas que desde la rigidez imponen unos protocolos inflexibles. Una escuela que ponga delante la necesidad vital de las personas, y en definitiva, aquello que las realiza y las hace felices. Algunos investigadores de la infancia (Tonucci, 2020) concluyen de sus indagaciones con el alumnado que las prioridades de la infancia tienen que ver con no tener que asistir a clases aburridas, con que su escuela no trate de las cosas que les importan, de sus sentimientos y de sus emociones, con que en su entornos escolares se permitan y se imponga actos de abuso y de violencia, con que sus maestros no luchen contra que se reproduzca el esquema social de los más fuertes, que les estigmatice y someta a través de relaciones machistas o les discrimine por razones de raza o de color de piel. En fin, parece que expresan que lo que verdaderamente es vital para ellos y ellas, no se trate ni se gestione en la escuela. En este sentido, piden a la escuela que conforme un currículo más rico en emociones, en experiencias, en escucha, en observación, en acompañamiento, en dedicación y en convivencia. En definitiva, la conformación de un repertorio de actuaciones que, de alguna forma, estaban relegadas del currículo oficial antes de la pandemia y que la propia crisis ha puesto de relevancia como imperiosas y necesarias para reducir el impacto de sus efectos. Por ello, se hace necesario aprovechar la salida de esta crisis para que la escuela

Revista Tópicos Educacionais, Pernambuco, v. 27, n. 01, p. 01-22, 2021. ISSN: 2448-0215. https://periodicos.ufpe.br/revistas/topicoseducacionais/index Dossiê "Conflitos, violências, bullying na escola: problemas da convivência potencializado pela pandemia?" DOI: 10.51359/2448-0215.2021.250402 
regrese a un espacio del que nunca debió marcharse, para centrarse en las verdaderas necesidades del alumnado reafirmando el compromiso y la actitud por esos valores que conforman lo que hemos denominado una pedagogía de cuidados (Avilés, 2020b).

Claro, esto plantea exigencias insoslayables a todos los miembros de la comunidad educativa, especialmente a quienes tienen un perfil educador: familias, profesorado y al propio alumnado que convive con sus colegas a diario. Supone salir de muchas zonas de confort, abandonar rutinas y pensar en tiempos, espacios y contenidos centrados en el bienestar, la observación, las emociones, la escucha, la sensibilidad, la motivación o la compensación de diferencias y vulnerabilidades. Y no es menos cierto, que aunque son necesarios, todavía son ámbitos poco transitados por los educadores, con carencias formativas y de intervención evidentes $\mathrm{y}$ con déficits de ubicación en los planes de estudio y organizativos y estructurales en la planificación de los centros, que no tienen definidos los marcos teóricos de actuación, las responsabilidades compartidas de actuación ni los mecanismos de detección y evaluación de necesidades ni los tiempos y espacios académicos de puesta en práctica. Por ello, la práctica docente, de forma progresiva, debe ir incorporando los cuidados en su pedagogía como una prioridad cuando se enfrenta a una alumna o alumno que percibe vulnerable, que escucha triste, que acude a la escuela con miedos o ,cuando se entrevista con una familia que encuentra desprovista de apoyo para enfrentar los efectos de la pandemia. Porque se han producido muchas carencias y pérdidas, y se deben abordar desde el cuidado emocional. Pérdidas que se muestran evidentes como las de vidas, de salud, de amistades, de vínculos, de empleo, de compañía, de alimentos. Pero también pérdidas más ocultas como las pérdidas en el aprendizaje, de confianza, de autoestima, en el acceso a fuentes de información o a redes sociales de apoyo o asistencia. Por tanto, en esas intenciones de cuidados la escuela debe tener activadas todas sus redes de detecciónprotección y su capacidad para visualizar todas las brechas que la pandemia está provocando (Avilés, 2020c), para no dejar a ninguna persona atrás. Pero en especial, la brecha emocional porque sus efectos se van a manifestar en las personas como exponente de estrés postraumático.

En esta tarea se destacan algunos ámbitos de trabajo esenciales por los que comenzar. En primer lugar, la escuela, cada maestra y maestro con su grupo de convivencia y de responsabilidad, tiene que prever y organizar un espacio-tiempo para tener un encuentro individual-colectivo con los suyos. Con su preparación, su liturgia, sus tiempos de habla, silencio y de escucha, de reflexión y participación. Se ha de escenificar un catarsis en que cada uno, una,

Revista Tópicos Educacionais, Pernambuco, v. 27, n. 01, p. 01-22, 2021. ISSN: 2448-0215. https://periodicos.ufpe.br/revistas/topicoseducacionais/index Dossiê "Conflitos, violências, bullying na escola: problemas da convivência potencializado pela pandemia?" DOI: $10.51359 / 2448-0215.2021 .250402$ 
voluntariamente, desde una posición de seguridad emocional pueda mostrar sus emociones y experiencias, lo que ha vivido y lo que ahora le atenaza o le preocupa. Estos momentos no tienen por qué ser únicos, se pueden establecer periódicamente con la finalidad de asegurar en el alumnado o en las familias que no están solos, que no sus pensamientos y emociones no son únicos, que en esto se camina como colectivo y el apoyo mutuo se tiene que asegurar. Estos escenarios y otros deben servir para plasmar el trabajo profesional de los cuidados.

Algunos se revelan imprescindibles, como el acompañamiento en los mensajes. Si algo ha provocado la pandemia han sido los momentos de confusión e incertidumbre. Especialmente el alumnado más pequeño, pero también el adolescente, se muestra necesitado de acompañamiento en sus pensamientos frente a lo que no entiende, se revela o confronta. Las situaciones límite o de máximo estrés o incertidumbre provocan pensamientos y concepciones inadecuadas e irracionales de la realidad en quienes las padecen, pero si son menores en formación y desarrollo, si cabe, su daño es mayor. El profesorado y los adultos que conviven con el alumnado deben ayudarles a estructurar sus mensajes respecto a lo sucede, cuando lo que enfrentan es incierto o no del todo comprensible. Esta necesidad es básica para evitar sufrimientos emocionales y deformaciones. El plano comunicativo es crucial porque el lenguaje exterior e interior no es inocuo. Los mensajes que producimos y que compartimos tienen efectos sobre las emociones. Se ha de ayudar al alumnado a construir mensajes constructivos y a evitar tanto los destructivos como los irracionales. El trabajo de análisis en colectividad puede dar confianza y aportar perspectivas nuevas. Esto ayudará construir un relato seguro y centrado sobre lo que sucede y que nos afecta como personas. Este relato comunicativo permitirá poner en juego en el profesorado o en las familias las herramientas cognitivas de que ya disponen como adultos al servicio de los menores, a quienes ayudará a estructurar el mensaje consistente y tranquilizador que necesitan. Mensajes y relato dan pie para practicar unas de las herramientas más útiles para la convivencia y para la prevención de las violencias como son diálogo y la escucha.

Por otra parte, los cuidados deben dirigirse también a lo vitalmente importante. La pandemia ha situado a muchas personas ante situaciones vitales difíciles o dolorosas. Las familias, el alumnado y el profesorado deben concederse tiempos para restañar las heridas y deben acompañar mutuamente las experiencias desde la presencia, la empatía, la ayuda y la compasión en muchos casos, esperando a que duelos y pérdidas se instalen en las memorias, logrando hacer prevalecer los recuerdos positivos a las evocaciones dolorosas.

Revista Tópicos Educacionais, Pernambuco, v. 27, n. 01, p. 01-22, 2021. ISSN: 2448-0215. https://periodicos.ufpe.br/revistas/topicoseducacionais/index Dossiê "Conflitos, violências, bullying na escola: problemas da convivência potencializado pela pandemia?" DOI: $10.51359 / 2448-0215.2021 .250402$ 
Lógicamente, pensar en una escuela así, es concebir la práctica educativa y la propia escuela como una institución que da respuesta también a las situaciones de emergencia en que están las personas, porque sus profesionales la conciben y la entienden como un espacio emocional y emocionante. En este sentido, la escuela es articulada como una comunidad de cuidados y como un espacio que puede y quiere curar. En ella, por tanto, se representan y se visualizan todas las necesidades y sus miembros están formados y están pendientes para observar e intervenir sobre las necesidades de los otros, como un elemento crucial y esencial de su tarea educativa y profesional.

En definitiva, pensar el espacio escolar y la comunidad educativa como un contexto en que los cuidados están organizados y presentes y se hacen intención de las relaciones interpersonales supone desarrollar unas herramientas para favorecer la convivencia y suponen un elemento preventivo crucial para evitar la manifestación de la violencia y su reproducción en formas de abuso, maltrato o imposición. Siempre hemos analizado por separado los tópicos entre convivencia en negativo y convivencia en positivo (Avilés, 2018c), que en investigación suelen centrar los esfuerzos de los estudiosos, pero esta diferenciación es meramente formal, dado que en la vida real se manifiesta interrelacionada y dependiente. Por eso, nuestra propuesta en los contextos escolares siempre ha sido ocuparse preventivamente de desarrollar elementos de convivencia positiva para que los efectos de estos programas y propuestas ayuden a evitar tener que ocupar la mayor parte del tiempo escolar en corregir de forma reactiva las manifestaciones negativas en la convivencia. Esta posición tiene el máximo respaldo por parte de diferentes propuestas pedagógicas preventivas que se llevan a cabo en distintos contextos escolares a partir de la Resolución Pacífica de los Conflictos (Boqué, 2009; Torrego, 2001), la puesta en práctica del modelo de Equipos de Ayuda (Avilés, Torres y Vián, 2008; Avilés, Cowie y Alonso, 2019) para prevenir el bullying, las Prácticas Restaurativas (Hansberry, 2016; Hopkins, 2004) como vías para salir de situaciones marcadas de daño o el Aprendizaje Servicio (Puig, 2010) como metodología para canalizar el compromiso cívico del alumnado y cubrir necesidades e interrelacionar las escuelas con sus contextos de forma proactiva.

Por tanto, ocuparse de la convivencia como intención y acción en los planes de las escuelas es apostar por reforzar la cohesión de los grupos que conviven en su seno, ayudarles a descentrarse de sus preocupaciones individuales y orientar sus miradas hacia las necesidades de las otras personas para identificarlas, comprenderlas y saber actuar cuando es adecuado hacerlo. Todo

Revista Tópicos Educacionais, Pernambuco, v. 27, n. 01, p. 01-22, 2021. ISSN: 2448-0215. https://periodicos.ufpe.br/revistas/topicoseducacionais/index Dossiê "Conflitos, violências, bullying na escola: problemas da convivência potencializado pela pandemia?" DOI: $10.51359 / 2448-0215.2021 .250402$ 
en conjunto supone un fortalecimiento de los vínculos relacionales y la generación de un clima escolar donde se hace posible compartir emociones y cuidar y ser cuidado.

\section{EL RETO DE LA CIBERCONVIVENCIA Y SU EDUCACIÓN}

Ciertamente, la pandemia ha forzado a la escuela a mirar de forma mucho más atenta sobre la tecnología y cómo integrarla en su cotidiano escolar y en las relaciones interpersonales. En este sentido, la escuela ya partía con desventaja en el abordaje de esta temática antes de la pandemia, pues aparecían insuficientemente desarrolladas algunas premisas básicas para el tratamiento del papel del uso de la tecnología en la propia escuela y en su currículo o las pautas educativas a trabajar con los escolares en su misma vida escolar y familiar y en el contexto de sus relaciones digitales entre pares. Así, no se contaba con su suficiente definición sobre el papel de las familias en la gestión digital, la integración curricular de las nuevas tecnologías en el currículo escolar y en la práctica docente, el uso adecuado o inadecuado de los dispositivos móviles en los espacios escolares, el protagonismo del alumnado como agentes educativos digitales entre sus iguales o sobre las pautas educativas digitales que se proporcionan desde los distintos agentes educativos para la gestión de la ciberconvivencia entre pares.

Por otra parte, ya hacía tiempo que en la convivencia escolar algunas de las manifestaciones de quiebra de la convivencia como el cyberbullying u otras formas de violencia, igual que las relaciones interpersonales en general, se hacían habituales en el plano digital, adoptando formas más crueles y espacios más difíciles de atajar (Avilés, 2013).

Con la llegada y el desarrollo de la pandemia y la extensión en la escuela de la educación a distancia o en remoto, el papel de los dispositivos electrónicos y las plataformas digitales han cobrado una relevancia evidente y se han generalizado en uso, marcando brecha en familias, alumnado y escuelas en el acceso a la información y a los recursos tecnológicos. Esto ha puesto de manifiesto una vez más las vulnerabilidades, pero además, ha situado de pleno las relaciones interpersonales entre pares y de familias y profesorado en el plano digital. Así, una necesidad educativa menos notoria antes de la pandemia, como era la intervención educativa en el plano de la ciberconvivencia, la pandemia la ha hecho más visible y más urgente de intervención a partir de pautas educativas comunes que ayuden a niños, niñas y adolescentes a conducirse en sus relaciones en las redes social y en el espacio digital como lo debían hacer en el espacio presencial. Por tanto, las redes sociales, internet y la manera en como los menores establecen sus relaciones en

Revista Tópicos Educacionais, Pernambuco, v. 27, n. 01, p. 01-22, 2021. ISSN: 2448-0215. https://periodicos.ufpe.br/revistas/topicoseducacionais/index Dossiê "Conflitos, violências, bullying na escola: problemas da convivência potencializado pela pandemia?" DOI: $10.51359 / 2448-0215.2021 .250402$ 
ellas son motivo educativo pendiente en la escuela de hoy. La ciberconvivencia es un escenario en que situar ya la intervención educativa de las comunidades educativas de forma prioritaria, puesto que es el lugar en que habitan nuestros escolares.

Sin embargo, la percepción que tienen sobre esta situación los agentes educativos que han de intervenir sobre ella ofrece algunos elementos de preocupación (Avilés, 2021 pág. 16):

- Cierto efecto indefensión en la comunidad educativa sobre la posibilidad o no de atajar real y educativamente el fenómeno.

- La preconcepción sobre una supuesta brecha generacional que entraría en contradicción con la cualificación o no de los adultos para influir positivamente en el menor en el uso seguro y responsable de la red (sentido común educativo), lo que les serviría como excusa y comodidad para no intervenir.

- La delegación de la intervención educativa sobre agentes de control y fuerza (las fuerzas de seguridad) en vez de sobre agentes propiamente educativos (profesorado, familias e iguales), con el mensaje que eso transmite y los efectos que provoca.

- La propia orientación de la intervención, desequilibradamente decantada sobre los riesgos y lo negativo de internet, con el rechazo adolescente que genera el mensaje, frente a planteamientos de oportunidades y prácticas positivas.

- La falta de programas educativos insertos en currículo con intención de intervenir en los procesos subyacentes a la toma de decisiones.

- El insuficiente protagonismo del alumnado en la intervención, sobre cómo perciben su experiencia vital en las tecnologías, sus formas de relación con terceros y de percepción del mundo por medio de las mismas.

Estos elementos son algunas de las principales preocupaciones a la hora de diseñar y llevar a término una intervención en ciberconvivencia. Por eso, hacen que cualquier actuación educativa no sirva para acometer un tema tan complejo, sensible y virtual como es la ciberconvivencia en los centros educativos. En este sentido, a continuación se proponen alternativas a estas preocupaciones marcando algunos principios que se han de asegurar y que deben guiar las líneas de intervención.

Revista Tópicos Educacionais, Pernambuco, v. 27, n. 01, p. 01-22, 2021. ISSN: 2448-0215. https://periodicos.ufpe.br/revistas/topicoseducacionais/index Dossiê "Conflitos, violências, bullying na escola: problemas da convivência potencializado pela pandemia?" DOI: $10.51359 / 2448-0215.2021 .250402$ 
A las dudas o incertidumbres que la comunidad educativa o cualquiera de sus sectores pueda albergar sobre su capacidad para abordar las situaciones de gestión de las relaciones interpersonales en las redes de sus propios hijos e hijas o de su alumnado, se ha de responder con propuestas educativas concretas que fomenten un trabajo colectivo y colaborativo en las comunidades educativas. No se puede hacer una intervención individual o segmentada. Los equipos de trabajo deben construir propuestas realistas, que partan de debates sinceros a partir de problemas reales, en que todos los miembros de la comunidad educativa estén representados y puedan participar y que permitan adoptar acuerdos y compromisos que ellos mismos puedan supervisar y modificar si es preciso.

Es cierto que las distintas generaciones no tienen una misma visión, ni destreza en el uso de las nuevas tecnologías o en los dispositivos móviles, ni suponen lo mismo para unos que para otros. Pero esto no significa que las distintas generaciones tengan distinto sentido común. De lo que se trata es de eso. Ayudar a los menores a conducirse con sentido común en las relaciones interpersonales en el ámbito digital. Se trata de la puesta en práctica de valores como el respeto a las otras personas, conductas de justicia para con uno mismo y las otras personas, miradas de empatía para entender lo que puede llegar a sentir el otro y ser solidario con sus necesidades o debilidades,... no son elementos técnicos que supongan especial actualización o entendimiento. Algunos autores constatan estos déficits de intermediación en las familias (Catalina y López de Ayala, 2013). Se trata de desarrollar pautas educativas y valores, que se expresan o no tanto en el plano presencial como en el digital. Por eso, los padres, madres y el profesorado deben hacer uso de ellos con los menores sin escusas de brecha generacional.

$\mathrm{Y}$ esto debe hacerse sin delegar en otros agentes que no tienen perfil educativo. Los agentes educativos son los legitimados. Desde una perspectiva autoritaria se puede pensar que un policía o un agente de seguridad pueden llegar a tener más eficacia en esto. Sin embargo, lo que se busca es educar, no atemorizar. El miedo no educa. Se pretende que el menor avance en su desarrollo moral hacia la autonomía moral (Kohlberg, 1992) y cuando está sentado ante una pantalla solo en su cuarto no hay ningún policía que le vigila para que se comporte conforme a la norma. La intervención educativa busca educar al menor, y por tanto, los agentes que mejor posicionados están para hacer esta tarea son sus iguales, sus padres y su profesorado. Son quienes pueden llegar a tener valor para ellos, los que pueden tener autoridad moral sobre él o ella y quienes habitualmente conviven o ciberconviven con él o ella. Por tanto, no son elementos ajenos

Revista Tópicos Educacionais, Pernambuco, v. 27, n. 01, p. 01-22, 2021. ISSN: 2448-0215. https://periodicos.ufpe.br/revistas/topicoseducacionais/index Dossiê "Conflitos, violências, bullying na escola: problemas da convivência potencializado pela pandemia?" DOI: $10.51359 / 2448-0215.2021 .250402$ 
a ellos que un día visitan el centro, les dan una charla y luego desaparecen. Por el contrario, les conocen, saben de sus gustos, miedos, costumbres y pueden intervenir educativamente con ellos de forma más conectada. Ciertamente, cada edad tiene referentes diferentes. Los más pequeños tienen en sus padres y madres los principales agentes educativos. Por eso, las familias deben acompañar a estos menores en el inicio del uso de las nuevas tecnologías y los dispositivos móviles. Deben aplicar sentido común a la hora de facilitarles esos dispositivos y valorar la necesidad de uso que tienen de ellos. En el acompañamiento, deben aportar criterio y supervisión en el abordaje de la privacidad de lo comunican, de cómo van a ir construyendo su identidad on line, sobre lo que comunican, a quién comunican y cómo lo comunican. En definitiva, un acompañamiento educativo que les aporte pautas para comportarse en la red y en las relaciones con otras personas. Por el contrario, los más mayores tienen a sus iguales como referentes entre quienes se miran, en ellos y en el grupo. De ahí, la importancia que en el seno de los grupo de clase, las escuelas dispongan de figuras entre el alumnado, como los cibermentores y cibermentoras (Avilés, 2018a), que actúen como referentes de conducta y mentoría y consejo en las redes. Por otra parte, esto solo es posible si la escuela y el profesorado lo promueve y lo organiza como otro Sistema de Apoyo entre Iguales (Avilés, 2018b) más entre el alumnado, porque llegue a la conclusión de que la ciberconvivencia también es un contenido propio de su trabajo educativo que planificar y en el que intervenir.

Por otra parte, resulta decisiva la orientación que se dé a la intervención en ciberconvivencia. Se ha de buscar un balance entre oportunidades y riesgos. La seguridad no debería suponer la restricción de oportunidades y la privación de las ventajas que tiene el mundo digital (Livingstone, 2013). No tiene ningún sentido demonizar las redes sociales o pensar que todo lo que hay y puede venir de internet es negativo para los escolares y sus relaciones. A ellos no solo les produce rechazo tal discurso, sino que desacredita a quienes lo proponen como referentes o como elementos de influencia. Además de no ser cierto, es poco efectivo educativamente. Los centros educativos deben encontrar en el tratamiento de la ciberconvivencia un equilibrio entre las oportunidades que ofrecen las relaciones interpersonales digitales y los riesgos que pueden producir. En este sentido, la orientación de los mensajes es importante. Relacionarse on line aporta cosas positivas, permite disfrutar y no sentirse solo, conocer, relacionarse, aprender, informarse, conectar y establecer amistades, influir, acceder a saberes, emociones y acciones positivas. Así mismo, trabajar en la escuela desde lo digital aporta perspectivas nuevas en el aprendizaje entre

Revista Tópicos Educacionais, Pernambuco, v. 27, n. 01, p. 01-22, 2021. ISSN: 2448-0215. https://periodicos.ufpe.br/revistas/topicoseducacionais/index Dossiê "Conflitos, violências, bullying na escola: problemas da convivência potencializado pela pandemia?" DOI: $10.51359 / 2448-0215.2021 .250402$ 
iguales, la práctica profesional, el uso de las redes de apoyo, la intercomunicación, la difusión de mensajes, la extensión de campañas, el enriquecimiento de los sistemas de participación, el pensamiento crítico o la promoción de valores.

También se ha de ser consciente de riesgos en las relaciones como el cyberbullying, el uso problemático, la adicción, la hiperconexión, la hipervirtualidad, la distancia emocional, el aislamiento social o la confusión fantasía-realidad. Son conocidos delitos como el cyberstalkiing, el sexting o el grooming, y de ellos los menores deben ser conocedores y utilizar prevenciones para defenderse de sufrirlos o provocarlos. Sin embargo, educarlos en elaborar criterios que les ayuden para tomar decisiones acertadas en sus relaciones interpersonales para sí mismos y para otras personas en internet y en las redes sociales, debería ser el objetivo de los programas de ciberconvivencia.

Y desafortunadamente esta es otra de las realidades sobre las que se debe trabajar. No existen muchos programas educativos que aborden la ciberconviencia de manera adecuada. Muchos de los programas educativos para trabajar la ciberconvivencia cargan las tintas más en lo negativo que en las oportunidades. Sin embargo, desde hace algún tiempo este enfoque más equilibrado se ha manifestado como más adecuado para ser trabajado en las escuelas (Vanderhoen, Schellens y Valcke, 2013). Algunos programas como el Programa PRIRES (Avilés, 2018a) plantea el trabajo en el seno de las escuelas a partir de actividades que están insertas en el Plan de Acción Tutorial de las mismas con el fin de no sea algo ajeno o postizo a la actividad escolar. Se desarrolla a lo largo de cincuenta y ocho sesiones que trata de manera sistemática tópicos educativos de ciberconvivencia referidos a bloques temáticos de privacidad, identidad digital, comunicación, empatía virtual, pensamiento consecuencial, autorregulación y prevención de riesgos. El Programa es llevado a cabo a lo largo cada curso distribuyendo las sesiones adaptadas a diferentes edades, de forma que a lo largo de la escolaridad el alumnado con su profesorado ha ido desarrollando esos tópicos. Por tanto, es el profesorado con las familias y el alumnado cibermentor quien lleva a la práctica el Programa por lo que es necesaria una formación previa con ellos.

$\mathrm{Y}$ es que para que la intervención en ciberconvivencia sea efectiva en las escuelas es preciso dar responsabilidad al alumnado, contar con los protagonistas de la misma, los alumnos y las alumnas. Ellos viven habitualmente en la red y ubican sus relaciones en el mundo virtual. Por lo tanto, es vital partir de sus experiencias y de lo que a diario viven en sus relaciones para poder plantear y construir respuestas ajustadas a los problemas que afrontan. Y en esas respuestas deben

Revista Tópicos Educacionais, Pernambuco, v. 27, n. 01, p. 01-22, 2021. ISSN: 2448-0215. https://periodicos.ufpe.br/revistas/topicoseducacionais/index Dossiê "Conflitos, violências, bullying na escola: problemas da convivência potencializado pela pandemia?" DOI: $10.51359 / 2448-0215.2021 .250402$ 
ser agentes protagonistas para que puedan responsabilizarse en compromisos adoptados de forma individual y colectiva de forma cada vez más madura y evolucionada. En esas respuestas, se han de incorporar las particularidades de sus formas de relación con terceros y la propia visión que del mundo tienen a través de la tecnología. En ese sentido, uno de los Sistemas de Apoyo entre Iguales (SAIs) como el de la Cibermentoría (Avilés y Ga Barreiro, 2016) es un buen ejemplo de asunción de las responsabilidades por parte del alumnado de la gestión de su propia ciberconvivencia.

\section{UNA TAREA DE LA COMUNIDAD EDUCATIVA EN SU CONJUNTO.}

Dar respuesta a las exigencias educativas que plantea una crisis como la que se está viviendo no es simple y abre diversos campos de actuación interrelacionados que exige de actuaciones intencionales, multidisciplinares y coordinadas. En este texto hemos reflexionado sobre algunas de las consecuencias de vulnerabilidad y desigualdad sobre la comunidad educativa, sobre los aprendizajes, la práctica docente, la convivencia, y en definitiva, sobre el bienestar de las personas que la componen. Sobre ellas se ha posicionado el foco de análisis y se han propuesto para cada uno de los sectores de la comunidad educativa líneas de intervención y cambio para la mejora de la escuela.

Al profesorado se le coloca en una situación de liderazgo pedagógico, y junto con la familia, funcionando como modelo de respuesta y de afrontamiento de situaciones. Se les confían tareas académicas y docentes, pero también de cuidados, de observación de necesidades, de acompañamiento, de asesoramiento crítico, de escucha, de compartición de tiempos..., y en terrenos como el de la ciberconvivencia van a intermediar y guiar las buenas prácticas comunicativas, las relaciones respetuosas, la empatía digital, la autorregulación o la capacidad de resiliencia.

A las familias les corresponde apoyar en todo momento a sus hijos e hijas, acompañándoles de forma guiada en el afrontamiento de las inseguridades y dificultades; participar de forma real en las escuelas, trabajando con lealtad educativa con el profesorado; estableciendo pautas educativas de supervisión con sus hijos e hijas, ajustando y no minimizando el impacto que situaciones de riesgo pueden llegar a ocasionar en quienes tienen a su cuidado, mediando en su crecimiento crítico ante lo que viven y observan, combinando autonomía y consistencia normativa.

Revista Tópicos Educacionais, Pernambuco, v. 27, n. 01, p. 01-22, 2021. ISSN: 2448-0215. https://periodicos.ufpe.br/revistas/topicoseducacionais/index Dossiê "Conflitos, violências, bullying na escola: problemas da convivência potencializado pela pandemia?" DOI: $10.51359 / 2448-0215.2021 .250402$ 
Al alumnado se le debe situar en su posición protagonista de su propia educación y del papel que pueden desempeñar con sus iguales. Son los primeros que auxilian y ayudan a los compañeros que están mal o son violentados. Ese dique de contención y salvaguarda emocional la escuela debe organizarlo y disponerlo para que funcione a máximo rendimiento desde el ejercicio de tareas de prevención en convivencia positiva. El potencial de conexión social que tienen de igual a igual (McLoughlin, Spears y Taddeo, 2018) debe ser una correa de transmisión donde discurran los valores más genuinos que fundamentan los derechos humanos de la ciudadanía, justicia, respeto, solidaridad, tolerancia, igualdad, ayuda, compasión, positividad y convivencia.

Ello será posible si se hace de forma conjunta, si desde cada sector se interviene de forma planificada, cooperativa e intencional, a través de planes de acción coordinados en las escuelas, trabajando las competencias educativas para la convivencia. Ojalá que a la salida de esta crisis podamos decir que estamos en condiciones de empezar a lograrlo, que la hemos aprovechado para mejorar la escuela que imaginamos y que queremos.

\section{REFERENCIAS BIBLIOGRÁFICAS}

Avilés, J. Ma. (2013). Análisis psicosocial del cyberbullying. Claves para una educación moral. Papeles del psicólogo, 34, (1), 65-73.

Avilés, J. M ${ }^{\mathrm{a}}$ (2018a). Educar en las redes sociales. Programa preventivo PRIRES. Bilbao: Desclée de Brouwer.

Avilés, J. Ma (2018b). Los Sistemas de Apoyo entre Iguales en la escuela. De los Equipos de Ayuda a la Cibermentoría. Americana: Adonis.

Avilés, J. M ${ }^{\mathrm{a}}$ (2018c). Convivir sin Bullying, compartiendo relaciones de respeto. Madrid: Narcea.

Avilés, J. Ma, Torres, N. y Vian, M.V. (2008). Equipos de ayuda, maltrato entre iguales y convivencia escolar. Electronic Journal of Research in Educational Psychology, 6, (3), 357376.

Avilés, J. Ma. (2019). Educar tras el Covid-19. Debate con gestores DEVIR. São Paulo: Devir.

Avilés, J. M (2020a). A pandemia da Covid-19 e a volta às aulas. Sao Paulo: DEVIR.

Avilés, J. Ma (2020b). Por una pedagogía de los cuidados. Sao Paulo: Red de Escuelas Brasileñas de la UNESCO. En línea: [https://www.sympla.com.br/encontro-nacional-das-escolasassociadas-da-unesco-no-brasil---2020__1044634, recuperado 8 de diciembre de 2020]

Avilés, J. M (2020c). El abuso emocional durante la pandemia. Barcelona: RIEEB.

Avilés, J. M M $^{\mathrm{a}}$ (2021). Educar para la ciberconvivencia en los centros educativos. Dirección y Liderazgo Educativo, 9, 16-20.

Avilés, J. M ${ }^{\mathrm{a}}$ y $\mathrm{G}^{\mathrm{a}}$ Barreiro, J. (2016, julio). Cibermentores. En línea: [http://convivenciaenlaescuela.es/, extraído el 16 de octubre de 2016]

Avilés, J. M., Torres, N. y Vian, M. V. (2008). Equipos de ayuda, maltrato entre iguales y convivencia escolar. Revista de Investigación Psicoeducativa, 6, 357-376.

Avilés, J.Ma , Cowie, H. y Alonso, MªN. (2019). El valor de la ayuda. Equipos de Ayuda frente al bullying. Barcelona: Horsori.

Revista Tópicos Educacionais, Pernambuco, v. 27, n. 01, p. 01-22, 2021. ISSN: 2448-0215.

https://periodicos.ufpe.br/revistas/topicoseducacionais/index

Dossiê "Conflitos, violências, bullying na escola: problemas da convivência potencializado pela pandemia?" DOI: $10.51359 / 2448-0215.2021 .250402$ 
Avilés, J.Ma, Aragao, A., Menin, S., Nunes, C. y Braga, A. (2020). Qual escola queremos após a pandemia. Campinas: Convivere Mais. En línea: [https://www.youtube.com/watch?v=nUCSiqH79dg, recuperado 30 de noviembre de 2020].

Bauman, Z. (2013). A cultura no mundo líquido moderno. Rio de Janeiro: Zahar Editores.

Boqué, Ma C. (2009). Construir la paz. Transformar los conflictos en oportunidades. Alicante: CAM.

Catalina, B. y López de Ayala, M.C. (2013). Los padres ante el uso de internet y redes sociales por menores. Control y protección. En Línea: [http://www.revistalatinacs.org/13SLCS/2013_actas.html. Recuperado 29/08/14]

Hansberry, B. (2016). Restorative practice in schools. Theory, skills and guidance. London: JKP Hopkins, B. (2004). Just Schools: A Whole School Approach to Restorative Justice, London: Jessica Kingsley.

Kohlberg, L. (1992). Psicologia del desarrollo moral. Bilbao: Desclée de Brouwer.

Latin American Council for Peace Research (2020). Manifiesto for a New Normality. Global campaign for PeacEducation: CLAIP.

Livingstone, S. (2013). Online risk, harm and vulnerability: Reflections on the evidence base for child Internet safety policy. Zer, 18(35), 13-28.

McLoughlin, L., Spears, B. y Taddeo, C. (2018). The importance of social connection for cybervictims: how connectedness and technology could promote mental health and wellbeing in young people. International Journal of Emotional Education, 10(1): 5-24.

Moreira, A. (2020). Lo que hemos aprendido en educación a causa de la crisis sanitaria. Educación 3.0. Recuperado de https://www.educaciontrespuntocero.com, 15 junio 2020.

Puig, J.M. (2010). Aprendizaje Servicio (ApS). Educación y compromiso cívico. Barcelona: Graó.

Reardon, B. (2020). Reviewing our pedagogy in walking the path to a new normality. Global campaing for PeacEducation: CLAIP

Tonucci, F.(2020). Reflexiones de la nueva era. El Diario de la Educación. Recuperado de https://www.eldiariodelaeducacion.com 19 junio 2020.

Torrego, J.C. (Ed.) (2001). Mediación de conflictos en instituciones educativas. Manual para la formación de mediadores. Narcea: Madrid.

Trujillo-Sáez, F., Fernández-Navas, M.; Montes-Rodríguez, M., Segura-Robles, A., AlaminosRomero, F.J. y Postigo-Fuentes, A.Y. (2020). Panorama de la educación en España tras la pandemia de COVID-19: la opinión de la comunidad educativa. Análisis cuantitativo: gráficos. Madrid: Fad. doi: 10.5281/zenodo-3878844

Vanderhoen, E., Schellens, T. y Valcke, M. (2013).Exploring the usefulness of school education out risks on social network sites: a survey study. En línea: [http://digitalcommons.uri.edu/cgi/viewcontent.cgi?article=1113\&context=jmle recuperado 05/02/2015].

Recebido em abril de 2021. Aprovado em junho de 2021.

Revista Tópicos Educacionais, Pernambuco, v. 27, n. 01, p. 01-22, 2021. ISSN: 2448-0215. https://periodicos.ufpe.br/revistas/topicoseducacionais/index Dossiê "Conflitos, violências, bullying na escola: problemas da convivência potencializado pela pandemia?" DOI: $10.51359 / 2448-0215.2021 .250402$ 